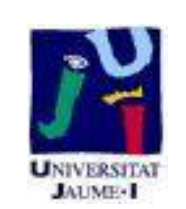

Título artículo / Títol article:

Jump state estimation with multiple sensors with packet dropping and delaying channels

Autores / Autors:

Dolz Algaba, Daniel ; Peñarrocha Alós, Ignacio ; Sanchis Llopis, Roberto

Revista:

International Journal of Systems Science

Versión / Versió:

Postprint

Cita bibliográfica / Cita bibliogràfica (ISO 690):

DOLZ, Daniel; PEÑARROCHA, Ignacio; SANCHIS, Roberto. Jump state estimation with multiple sensors with packet dropping and delaying channels. International Journal of Systems Science, 2014, no aheadof-print, p. 1-12.

url Repositori UJ:

http://repositori.uii.es/xmlui/handle/10234/134925 
To appear in the International Journal of Systems Science

Vol. 00, No. 00, Month 20XX, 1-18

\title{
Jump state estimation with multiple sensors with packet dropping and delaying channels.
}

\author{
Daniel Dolz, Ignacio Peñarrocha* and Roberto Sanchis \\ Departament d'Enginyeria de Sistemes Industrials i Disseny, Universitat Jaume I, Casetelló, Spain
}

(Received 00 Month 20XX; final version received 00 Month 20XX)

\begin{abstract}
This work addresses the design of a state observer for systems whose outputs are measured through a communication network. The measurements from each sensor node are assumed to arrive randomly, scarcely and with a time-varying delay. The proposed model of the plant and the network measurement scenarios cover the cases of multiple sensors, out-of-sequence measurements, buffered measurements on a single packet and multi-rate sensor measurements. A jump observer is proposed, that selects a different gain depending on the number of periods elapsed between successfully received measurements and on the available data. A finite set of gains is precalculated off-line with a tractable optimization problem, where the complexity of the observer implementation is a design parameter. The computational cost of the observer implementation is much lower than in the Kalman filter, whilst the performance is similar. Several examples illustrate the observer design for different measurements scenarios and observer complexity and show the achievable performance.
\end{abstract}

Keywords: State estimation; Packet dropouts; Networked control systems; Wireless sensor network; Stochastic stability; Packet-delaying networks; Out-of-sequence measurements (OOSMs); Multisensor systems; Bufferized measurement

\section{Introduction}

In the last years many processes in industry are controlled or supervised through sensors, actuators and controllers connected to a shared network (wired or wireless) (see Gupta and Chow (2010)). The use of a network causes packet dropout, delays or intermittent partial observations when acquiring data from sensors, and the control and estimation through a network must overcome these problems.

The Kalman filter tackles the state estimation over networks problem updating the observer gain at each sampling time with the corresponding observation matrix. This approach leads to a time varying gain that must be computed online even for linear time invariant systems (e.g. Sinopoli et al. (2004); Schenato $(2007,2008)$ ). The computational cost of the online implementation is unaffordable for some applications, what motivates the search for computationally low cost alternatives.

The use of precalculated gains reduces the implementation cost in terms of computing capacity, but increases the estimation error and requires both storage and a mechanism to choose the appropriate gain at each sampling time (e.g. Smith and Seiler (2003); Sahebsara et al. (2007); Peñarrocha et al. (2012)). A constant gain approach leads to the lower storage requirement but also to the lower performance. The jump linear estimator approach (Fletcher et al. (2006)) improves the estimation with a set of precalculated gains that are used at each sampling time depending on the actual available measurements, requiring both storage and the implementation

*Corresponding author. Email: ipenarro@uji.es 
of a selection algorithm. If the set of gains is also a function of the history of measurement availabilities (called finite loss history estimator in Smith and Seiler (2003)) a better performance is achieved at the cost of more implementation complexity in the selection of the appropriate gain. An intermediate approach in terms of storage and selector complexity consists of a dependency on the actual available measurements and on the number of consecutive dropouts since last available measurement (Peñarrocha et al. (2012); Peñarrocha et al. (2014)).

Computing the gains off-line requires prior knowledge about the network dropout. When the network behaves as a Markov chain, the design uses the transition probabilities (Smith and Seiler (2003)), and when the Markov chain is irreducible and aperiodic (i.e., Bernoulli distribution), the reception probabilities (stationary distribution of the Markov chain) give enough information for design or analysis purposes (Sinopoli et al. (2004)). Under unknown statistic information and scarce measurements, the maximum possible number of consecutive dropouts, if it is assumed to be bounded, gives useful information for the design (Peñarrocha et al. (2012)).

In this paper we propose a jump linear estimator that leads to low cost implementation and acceptable performance for networks with Bernoulli distributions. The gains depend on the combination of actual available measurements and on the number of consecutive periods without data since the last data arrived. As that number is unbounded for Bernoulli distributions, we propose to use a constant gain when the number of consecutive dropouts exceeds a given threshold, and derive an expression to determine the effect of this threshold on the achievable performance. Furthermore, we propose to reduce the number of stored gains by means of sharing the use of each gain for different combinations of available measurements.

The main difference with the work Smith and Seiler (2003) is an important reduction in the number of gains and in the complexity of the gain selection mechanism under scarce measurements, while leading to a similar performance. With respect to work Peñarrocha et al. (2012), the main differences are that we do not need to assume that the number of consecutive packet dropouts is bounded, and that we do not discard the measurements from which the state is not detectable. Other differences with those previous works are the integration of delayed measurements and irregular sampling scenarios as out-of-sequence or buffered measurements, and the proposal of different strategies to reduce the complexity of the observer in order to find a compromise between implementation cost and performance.

The paper has the following structure: in Section 2 we describe the process, present the observer algorithm and derive the estimation error. In Section 3 we develop the convex optimization based observer design, and demonstrate the convergence of the estimator. In section 5 we propose some possible scenarios groupings to find a compromise between implementation cost and performance. In Section 6 several examples show the validity of the proposal, compared to the Kalman filter approach. Finally, the main conclusions are summarized in Section 7.

\section{Problem approach}

Let us assume a linear time invariant discrete time system defined by equations

$$
x[t+1]=A x[t]+B_{u} u[t]+B_{w} w[t]
$$

where $x \in \mathrm{R}^{n}$ is the state, $u \in \mathrm{R}^{n_{u}}$ is the input, and $w \in \mathrm{R}^{n_{w}}$ is the state disturbance, assumed as a white noise signal of zero mean and known covariance $\mathcal{E}\left\{w[t] w[t]^{T}\right\}=W$. Let us assume that samples from several sensors are taken synchronously with the input update but received through a network with packet dropouts and induced time-varying delays. We denote as $t_{k}$ the instant of sample reception, and define the measurement value by

$$
m_{i, k}=c_{i, k} x\left[t_{k}-d_{i, k}\right]+v_{i, k}, \quad i=1, \ldots, n_{m}
$$


where $m_{i, k}$ represents the $k$-th measurement of sensor $i$, and $v_{i, k}$ the sensor noise on the $k$-th sample, assumed an independent zero mean white noise signal with known variance $\mathcal{E}\left\{v_{i, k}^{2}\right\}=\sigma_{i}^{2}$. We assume synchronization between sensors and the estimator unit and time-tagged message sending, that allows us to know the transmission delay. $d_{i, k} \in \mathrm{N}$ is the network induced delay measured in number of periods. We introduce an extended order model to avoid running backwards the model when dealing with delayed measurements. We take into account in the model delays up to a value $\bar{d} \in \mathrm{N}$ that must be selected in the estimator design procedure.

Remark 2.1. In the observer to be designed we discard measurements with a delay higher than $\bar{d}$. Note that $\bar{d}$ is not a network parameter (the network could have unbounded delays), but an observer design parameter (see Fig.2 in Schenato (2008)). As $\bar{d}$ may be lower than the bound on the real delays, reducing $\bar{d}$ may decrease the probability of having available measurements, but will also reduce the model order and hence, the complexity of the estimator. For a given delay distribution, the selection of $\bar{d}$ is a tradeoff between estimator complexity (related to the order) and achievable performance (related to the amount of available data used by the estimator to be designed). Example 6.2 illustrates this compromise in the choice of $\bar{d}$.

The model including the delayed states is

$$
\bar{x}[t+1]=\bar{A} \bar{x}[t]+\bar{B}_{u} u[t]+\bar{B}_{w} w[t],
$$

where $\bar{x}[t+1]=\left[x[t+1]^{T} \cdots x[t-\bar{d}+1]^{T}\right]^{T}$ and

$$
\bar{A}=\left[\begin{array}{cccc}
A & 0 & \cdots & 0 \\
I & \cdots & 0 & \vdots \\
\vdots & \ddots & \vdots & \vdots \\
0 & \cdots & I & 0
\end{array}\right], \quad \bar{B}_{u}=\left[\begin{array}{c}
B_{u} \\
0
\end{array}\right], \quad \bar{B}_{w}=\left[\begin{array}{c}
B_{w} \\
0
\end{array}\right]
$$

with the measurement equation

$$
m_{i}\left[t_{k}\right]=\underbrace{\left[0_{1 \times(n) d_{i, k}} c_{i} 0_{1 \times(n)\left(\bar{d}-d_{i, k}\right)}\right]}_{c_{i}\left[t_{k}\right]} \bar{x}\left[t_{k}\right]+v_{i, k} .
$$

As $c_{i}\left[t_{k}\right]$ can take $\bar{d}+1$ different values (depending on the delay $d_{i, k}$ ), we enumerate with $j \in\left[1, n_{m}(1+\bar{d})\right]$ each combination of sensor $i=1, \ldots, n_{m}$ plus possible delay $d=0, \ldots, \bar{d}$, and consider each one of those combinations as if they were measurements from different (fictitious) sensors. The enumeration we choose follows the law $j=(i-1)(1+\bar{d})+(d+1)$, and we express the measurement equations of the fictitious sensors $j$ with a constant output matrix as

$$
\bar{m}_{j}\left[t_{k}\right]=\bar{c}_{j} \bar{x}\left[t_{k}\right]+\bar{v}_{j, k}, \quad j=1, \ldots, \bar{n}_{m}
$$

with $\bar{n}_{m}=n_{m}(1+\bar{d})$ the number of total (real and fictitious) sensors, and

$$
\bar{c}_{j}=\left[0_{1 \times(n) d} c_{i} 0_{1 \times(n)(\bar{d}-d)}\right] .
$$

This notation allows us to deal with out-of-sequence received measurements, with received packets including measurements from one sensor sampled on different instants (i.e., buffered measurements), and avoids the use of time varying matrices $c_{i}\left[t_{k}\right]$.

We propose the following state estimation algorithm. At each control period, the model is run 
in open loop leading to

$$
\hat{\bar{x}}\left[t^{-}\right]=\bar{A} \hat{\bar{x}}[t-1]+\bar{B}_{u} u[t-1] .
$$

If no measurement is received, the best estimation of the system state is the open loop one, i.e., $\hat{\bar{x}}[t]=\hat{\bar{x}}\left[t^{-}\right]$. If some message arrives at time $t=t_{k}$, the state is updated as

$$
\hat{\bar{x}}\left[t_{k}\right]=\hat{\bar{x}}\left[t_{k}^{-}\right]+\sum_{j=1}^{\bar{n}_{m}} l_{j, k}\left(\bar{m}_{j, k}-c_{j} \hat{\bar{x}}\left[t_{k}^{-}\right]\right),
$$

where $l_{j, k}$ is the updating gain that applies to the $k$-th sample of sensor $j$ if available at the estimator node. The design of $l_{j, k}$ is one of the main concerns of this work and it will be discussed later in detail.

Let us define the sensor availability factor $\alpha_{j}[t]$ of the $j$-th sensor $\left(j=1, \ldots, \bar{n}_{m}\right)$ for every control period as

$$
\alpha_{j}[t]=\left\{\begin{array}{l}
0 \text { when } \bar{m}_{j} \text { is lost in } t \\
1 \text { when } \bar{m}_{j} \text { is received in } t
\end{array}\right.
$$

Let us define the availability matrix $\operatorname{as}^{1} \alpha[t]=\bigoplus_{j=1}^{\bar{n}_{m}} \alpha_{j}[t]$, that is a binary diagonal matrix with ones only at positions $j j$ such that $\alpha_{j}[t]=1$. Using this matrix, we rewrite the received measurements at instant $t$ as $\bar{m}[t]=\alpha[t](\bar{C} \bar{x}[t]+\bar{v}[t])$, with $\bar{m}[t]=\left[\bar{m}_{1}[t] \cdots \bar{m}_{\bar{n}_{m}}[t]\right]^{T}$, $\bar{v}_{k}=$ $\left[\bar{v}_{1}[t] \cdots \bar{v}_{\bar{n}_{m}}[t]\right]^{T}$, where a null value is assumed when a measurement is lost, the rows of $\bar{C}$ are $\bar{c}_{j}$, and where $\bar{v}[t]$ is the measurement noise vector with covariance

$$
\mathcal{E}\left\{\bar{v}[t] \bar{v}[t]^{T}\right\}=V=\bigoplus_{i=1}^{n_{m}}\left(\bigoplus_{j=1}^{1+\bar{d}} \sigma_{i}^{2}\right)
$$

assuming a non correlated noise. A control period in which all information from sensors is lost leads to $\alpha[t]=0$. If at a given control period all the information from each sensor is available, then $\alpha[t]=I$ (as we assume delayed measurements this means an arrival of a packet with information of each sensor from $t-\bar{d}$ to $t$ ).

With the previous notation, and considering a null value on the unavailable measurements, we rewrite the update equation (7) as

$$
\hat{\bar{x}}\left[t_{k}\right]=\hat{\bar{x}}\left[t_{k}^{-}\right]+L_{k} \alpha_{k}\left(\bar{m}_{k}-\bar{C} \hat{\bar{x}}\left[t_{k}^{-}\right]\right),
$$

where $L_{k}$ is the updating matrix. Defining the estimation error at the updating periods as $\tilde{x}\left[t_{k}\right]=\bar{x}\left[t_{k}\right]-\hat{\bar{x}}\left[t_{k}\right]$, the estimation error dynamic is

$$
\begin{aligned}
\tilde{x}\left[t_{k}\right]= & \left(I-L_{k} \alpha_{k} \bar{C}\right)\left(\bar{A}^{N_{k}} \tilde{x}\left[t_{k-1}\right]+\sum_{i=0}^{N_{k}-1} \bar{A}^{i} \bar{B}_{w} w\left[t_{k-1}+i\right]\right) \\
& -L_{k} \alpha_{k} \bar{v}_{k},
\end{aligned}
$$

where $N_{k}$ denotes the number of consecutive periods without measurements, i.e., $N_{k}=t_{k}-t_{k-1}$. To obtain equation (11) we firstly run the estimator (6) in open loop from $t_{k-1}$ to $t_{k}$ and apply the update equation (10), secondly we run the model (3) from $t_{k-1}$ to $t_{k}$, and finally we substract the two results (see Peñarrocha et al. (2012)).

${ }^{1} \oplus$ denotes the direct sum of matrices 
Each combination of available measurements leads to a different value of matrix $\alpha[t]$ at each control period. These values are within a known set

$$
\alpha[t] \in \Xi=\left\{\eta_{0}, \eta_{1}, \ldots, \eta_{r}\right\}
$$

where $\eta_{i}$ denotes each possible combination (sampling scenario). In the general case, any combination of available sensor measurements and delays is possible, leading to $r=2^{\bar{n}_{m}}-1$. Matrix $\eta_{0}$ denotes the scenario without available measurements, i.e., $\eta_{0}=0$, and the set of scenarios including some available measurement is denoted as $\Xi=\Xi \backslash \eta_{0}=\left\{\eta_{1}, \ldots, \eta_{r}\right\}$.

Remark 2.2. In each real application, the set $\Xi$ gathers the possible cases, and becomes a parameter design if the sensors have some processing capabilities. If the sensor nodes collect measurements on a buffer of size $\bar{b}$ and transmit the full buffer every period (as in works Schenato (2008); Moayedi et al. (2011); Zhu et al. (2012)), then each $\eta_{i}$ will have ones in the positions related to the sensors from which the gathered delayed measurements are sent, if they are received for the first time and their associated delay is lower than $\bar{d}$. In the multi-rate approach of Liang et al. (2010), each $\eta_{i}$ will represent the possible measurement combinations in the global period.

The probability of each case on $\Xi$ characterizes the network and is the prior knowledge that allows us to make a design with stochastic properties. Let us denote the probability of having a given set of measurements at period $t$ as $p_{i}=\mathcal{P}\left\{\alpha[t]=\eta_{i}\right\}$, where $p_{0}$ denotes the probability of having no measurements. Once a packet with data from several sensors arrives at $t_{k}$, the probability of having $N-1$ consecutive periods without data is given by

$$
\mathcal{P}\left\{\bigcap_{t=t_{k}+1}^{t_{k}+(N-1)} \alpha[t]=\eta_{0}\right\}=p_{0}^{N-1} .
$$

Remark 2.3. If the arrival from each sensor with a given delay is an independent and identically distributed process (as in Wang et al. (2012); Wu et al. (2012); Jia et al. (2012)), we compute the probabilities of each sampling scenario as follows. Let us denote as $\beta_{j}$ the probability of a measurement from fictitious sensor $j$ being available at a given control period, i.e. $\beta_{j}=\mathcal{P}\left\{\alpha_{j}[t]=\right.$ $1\}$. The complementary probability of failing on receiving an output sample from sensor $j$ is $\mathcal{P}\left\{\alpha_{j}[t]=0\right\}=1-\beta_{j}$, and the probability of having no measurement available at a given control period is

$$
p_{0}=\mathcal{P}\left\{\alpha[t]=\eta_{0}\right\}=\prod_{j=1}^{\bar{n}_{m}}\left(1-\beta_{j}\right) .
$$

The probability of having some sensor available is $\mathcal{P}\{\alpha[t] \in \bar{\Xi}\}=1-p_{0}$, and the probability of having a given combination of available sensors $\eta_{i} \in \bar{\Xi}$ is

$$
p_{i}=\mathcal{P}\left\{\alpha[t]=\eta_{i}\right\}=\prod_{\substack{j=1 \\ \forall \eta_{i, j}=0}}^{\bar{n}_{m}}\left(1-\beta_{j}\right) \prod_{\substack{j=1 \\ \forall \eta_{i, j}=1}}^{\bar{n}_{m}} \beta_{j}, i=1, \ldots, r
$$

where $\eta_{i, j}$ refers to the $j$-th diagonal entry of $\eta_{i}$.

The aim of this work is to compute the gain matrices $L_{k}$ that minimize the state estimation error in the mean square sense requiring low computing and storage capabilities (see Section 3). We first differentiate the computational cost of online and offline gains computing, and then differentiate the storage requirements between precomputed gains approaches. 
Using a Kalman filter Schenato (2008) leads to a time varying gain $L_{k}$ whose online implementation requires $2 \bar{n}^{3}+\bar{n}^{2} n_{w}+\bar{n} n_{w}^{2}+4 \bar{n}^{2} \bar{n}_{m}+2 \bar{n} \bar{n}_{m}^{2}+5 \bar{n}_{m}^{3}+2 \bar{n}^{2}+\bar{n} n_{u}+2 \bar{n} \bar{n}_{m}+\bar{n}_{m}^{2}+2 \bar{n}+\bar{n}_{m}$ (where $\bar{n}=n(\bar{d}+1)$ ) floating-point operations (FLOPs) per period, leading to high computation requirements and to possible numerical problems due to the inversion of a matrix of at most $\bar{n}_{m} \times \bar{n}_{m}$. Using a jump linear estimator with a finite set of stored gains leads to $\bar{n}^{2}+\bar{n} n_{u}+2 \bar{n} \bar{n}_{m}+2 \bar{n}+\bar{n}_{m}$ FLOPs per period, that is much lower than the previous one.

In the motivation example in Smith and Seiler (2003), the authors show that the gains obtained with a Kalman filter depend on the history of combination of sensor availability. From this observation they propose a finite history jump linear estimator whose gains depend on $\alpha[t], t=$ $t_{k}-h, \ldots, t_{k}$, and chose a history bound $h$, what requires storing $\left(2^{\bar{n}_{m}}-1\right) 2^{\bar{n}_{m}(h-1)}$ gains. In that motivation example, the gains depend also strongly on the number of consecutive periods without data between receptions $\left(N_{k}\right)$. From that observation, we propose a jump linear estimation that depends both on $N_{k}$ and $\alpha_{k}$ and stores at most $\left(2^{\bar{n}_{m}}-1\right) \bar{N}$ gains, with $\bar{N}$ our history bound that must be chosen as a compromise between observer complexity (number of stored gains) and achievable performance. We define the gains as follow

$$
L_{k}= \begin{cases}L_{N, i} & \text { if } N_{k}=N<\bar{N} \text { and } \alpha_{k}=\eta_{i} \\ L_{\bar{N}, i} & \text { if } N_{k} \geq \bar{N}, \text { and } \alpha_{k}=\eta_{i}\end{cases}
$$

for $\alpha_{k}=\eta_{1}, \ldots, \eta_{r}$. The matrices are computed off-line leading to the set of matrices

$$
L_{k} \in \mathcal{L}=\left\{L_{1,1}, \ldots, L_{1, r}, \ldots, L_{\bar{N}, r}\right\}
$$

that will be used to implement the jump linear estimator.

Remark 2.4. In this work, $N_{k}$ is assumed to be unbounded and $\bar{N}$ is a tuning parameter that affects to the number of stored gains, but it is not a parameter that describes the network behaviour. In Sanchis et al. (2007); Peñarrocha et al. (2012) the nodes are assumed to guarantee somehow that $N_{k}$ remains below $\bar{N}=\max \left\{N_{k}\right\}$, where $\bar{N}$ defines both the network behavior and the number of stored gains.

\section{Observer design}

In the following theorem we obtain the recursion that defines the evolution of the covariance matrix of the state estimation error. We will use this result later to compute the observer gains.

Theorem 3.1. Let $P_{k-1}=\mathcal{E}\left\{\tilde{x}_{k-1} \tilde{x}_{k-1}^{T}\right\}$ be the covariance matrix for the state estimation error updated at the measurement instant $t_{k-1}$. Then, the expected value of the covariance matrix at the measurement instant $t_{k}$ is given by

$$
\begin{aligned}
& \mathcal{E}\left\{P_{k}\right\}=\sum_{N=1}^{\bar{N}-1} p_{0}^{N-1} \sum_{i=1}^{r} p_{i} L_{N, i} \eta_{i} V \eta_{i}^{T} L_{N, i}^{T} \\
& +\frac{p_{0}^{\bar{N}-1}}{1-p_{0}} \sum_{i=1}^{r} p_{i} L_{\bar{N}, i} \eta_{i} V \eta_{i}^{T} L_{\bar{N}, i}^{T} \\
& +\sum_{N=1}^{\bar{N}-1} p_{0}^{N-1} \sum_{i=1}^{r} p_{i} F_{N, i}\left(\bar{A}^{N} P_{k-1}\left(\bar{A}^{N}\right)^{T}+S_{W, N}\right) F_{N, i}^{T} \\
& +\sum_{i=1}^{r} p_{i} F_{\bar{N}, i}\left(p_{0}^{\bar{N}-1} \bar{A}^{\bar{N}} S_{P, k-1}\left(\bar{A}^{\bar{N}}\right)^{T}+\bar{S}_{W}\right) F_{\bar{N}, i}^{T}
\end{aligned}
$$


where $S_{P, k-1}=\sum_{i=0}^{\infty} p_{0}^{i} \bar{A}^{i} P_{k-1}\left(\bar{A}^{i}\right)^{T}$, expressed as

$$
S_{P, k-1}=\operatorname{vec}^{-1}\left(\left(I-p_{0} \bar{A} \otimes \bar{A}\right)^{-1} \operatorname{vec}\left(P_{k-1}\right)\right)
$$

fulfills $S_{P, k-1}-p_{0} \bar{A} S_{P, k-1} \bar{A}^{T}=P_{k-1}$, and

$$
\begin{aligned}
& F_{N, i}=I-L_{N, i} \eta_{i} \bar{C}, \\
& S_{W, N}=\sum_{j=1}^{N} \bar{A}^{j-1} \bar{B}_{w} W \bar{B}_{w}^{T}\left(\bar{A}^{j-1}\right)^{T}, \\
& \bar{S}_{W}=\frac{p_{0}^{\bar{N}-1}}{1-p_{0}}\left(S_{W, \bar{N}}+p_{0} \bar{A}^{\bar{N}} S_{W, \infty}^{\prime}\left(\bar{A}^{\bar{N}}\right)^{T}\right), \\
& S_{W, \infty}^{\prime}=\operatorname{vec}^{-1}\left(\left(I-p_{0} \bar{A} \otimes \bar{A}\right)^{-1} \operatorname{vec}\left(\bar{B}_{w} W \bar{B}_{w}^{T}\right)\right) .
\end{aligned}
$$

Proof. Let us assume that at $t=t_{k-1}$ a new measurement is available and the state estimation is updated with equation (7) leading to a covariance matrix for the estimation error $\mathcal{E}\left\{\tilde{x}\left[t_{k-1}\right] \tilde{x}\left[t_{k-1}\right]^{T}\right\}=P_{k-1}$. The expected value of the covariance matrix for the estimation error at the next update period is

$$
\begin{aligned}
& \mathcal{E}\left\{P_{k}\right\}=\mathcal{E}\left\{\left(I-L_{k} \alpha_{k} \bar{C}\right) \bar{A}^{N_{k}} P_{k-1}\left(\bar{A}^{N_{k}}\right)^{T}\left(I-L_{k} \alpha_{k} \bar{C}\right)^{T}\right\} \\
& +\mathcal{E}\left\{\left(I-L_{k} \alpha_{k} \bar{C}\right) \sum_{i=0}^{N_{k}-1} \bar{A}^{i} \bar{B}_{w} W \bar{B}_{w}^{T}\left(\bar{A}^{i}\right)^{T}\left(I-L_{k} \alpha_{k} \bar{C}\right)^{T}\right\} \\
& +\mathcal{E}\left\{L_{k} \alpha_{k} V \alpha_{k} L_{k}^{T}\right\}
\end{aligned}
$$

considering the independency between $x\left[t_{k-1}\right], v_{k}$ and $w\left[t_{k-1}+i\right]$ for $i=0, \ldots, N_{k}-1$, and assuming $w[t]$ an uncorrelated noise. We express the different expectations considering the probability of the number of intersample periods $N_{k}$, the gain matrix dependency (16), and the rule of average conditional expectations. After algebraic computation and variable substitution it follows (18).

The previous theorem shows a recursion on the covariance matrix that we write $P_{k}=\mathfrak{E}\left\{P_{k-1}\right\}$, being $\mathfrak{E}\left\{P_{k-1}\right\}$ the linear operator that returns the right hand of equation (18). In order to compute the observer gains off-line, one must find the stable solution to equation $\mathfrak{E}\left\{P_{k-1}\right\}=$ $P_{k-1}$, but the relationship between $S_{P, k-1}$ and $P_{k-1}$ in (19) prevents from using standard Riccati solvers. We compute the set of gains $\mathcal{L}$ solving the optimization problem ${ }^{2}$

$$
\min _{\mathcal{L}, P} \operatorname{tr}(P), \quad \text { s.t. } \mathfrak{E}\{P\}-P \preceq 0,
$$

that allows us to include different constraints on the set of gains to reduce the observer complexity. We present the following numerical solution to this problem based on linear matrix inequalities and bilinear equality constraints that makes it easy to include different constraints over observer gains.

${ }^{2} A \preceq 0$ means that matrix $A$ is negative semidefinite, and $A \preceq B$ means that $A-B$ is negative semidefinite. Similar applies to $\prec, \succ$ and $\succeq$. 
Theorem 3.2. If there exist matrices $P, Q, R$ and a set of matrices $\mathcal{L}=\left\{L_{1,1}, L_{1, r}, \ldots, L_{\bar{N}, r}\right\}$ such that

$$
\begin{aligned}
& {\left[\begin{array}{cccc}
P & \bar{M} \overline{\bar{A}} \bar{M} \bar{W} & \bar{X} \bar{V} \\
\overline{\bar{A}}^{T} \bar{M}^{T} & \bar{Q} & 0 & 0 \\
\bar{W}^{T} \bar{M}^{T} & 0 & \bar{W} & 0 \\
\bar{V}^{T} \bar{X}^{T} & 0 & 0 & \bar{V}
\end{array}\right] \succeq 0,} \\
& P Q=I, S_{P} R=I,
\end{aligned}
$$

with ${ }^{3}$

$$
\begin{aligned}
& S_{P}=\operatorname{vec}^{-1}\left(\left(I-p_{0} \bar{A} \otimes \bar{A}\right)^{-1} \operatorname{vec}(P)\right), \\
& \bar{X}=\left[\begin{array}{lll}
\sqrt{p_{1}} L_{1,1} \eta_{1} & \cdots \sqrt{p_{1}} L_{1, r} \eta_{r} \cdots \sqrt{p_{N}} L_{\bar{N}, r} \eta_{r}
\end{array}\right], \\
& \bar{M}=\left[\begin{array}{lll}
M_{1} & \cdots & M_{\bar{N}}
\end{array}\right], M_{N}=\left[\begin{array}{lll}
\sqrt{p_{1}} F_{N, 1} & \cdots & \sqrt{p_{r}} F_{N, r}
\end{array}\right] \text {, } \\
& \overline{\bar{A}}=\bigoplus_{N=1}^{\bar{N}} p_{0}^{N-1}\left(\bigoplus_{i=1}^{r} \bar{A}^{N}\right), \\
& \bar{W}=\left(\bigoplus_{N=1}^{\bar{N}-1} p_{0}^{N-1}\left(\bigoplus_{i=1}^{r} S_{W, N}\right)\right) \oplus\left(\bigoplus_{i=1}^{r} \bar{S}_{W}\right), \\
& \bar{V}=\left(\bigoplus_{N=1}^{\bar{N}-1} p_{o}^{N-1}\left(\bigoplus_{i=1}^{r} V\right)\right) \oplus\left(\bigoplus_{i=1}^{r} \frac{p_{0}^{\bar{N}-1}}{1-p_{0}} V\right), \\
& \bar{Q}=\left(\bigoplus_{N=1}^{\bar{N}-1} p_{o}^{N-1}\left(\bigoplus_{i=1}^{r} Q\right)\right) \oplus\left(\bigoplus_{i=1}^{r} p_{0}^{\bar{N}-1} R\right) .
\end{aligned}
$$

then, recursion $\mathfrak{E}\{\cdot\}$ over $P$ fulfills $\mathfrak{E}\{P\}-P \preceq 0$. Furthermore, the optimization problem

$$
\min _{P} \operatorname{tr}(P) \text { subject to }(25),(26)
$$

is a solution of (24).

Proof. Applying extended Schur complements on (25) and taking into account (26) makes problem (24) and (27) equivalent.

We show next that if we apply the gains $\mathcal{L}$ obtained from problem $(27)$, then the sequence $\left\{P_{k}\right\}$ converges to the unique solution $P$ obtained in (27). Let us first introduce the following lemma borrowed from Sinopoli et al. (2004).

Lemma 3.1. Define the linear operator

$$
\mathfrak{L}(Y)=\sum_{N=1}^{\bar{N}-1} p_{0}^{N-1} \sum_{i=1}^{r} p_{i} F_{N, i} \bar{A}^{N} Y\left(\bar{A}^{N}\right)^{T} F_{N, i}^{T}
$$

\footnotetext{
3 Operator $\operatorname{vec}(A)$ generates a vector by stacking the columns of matrix $A$. Operator $v e c^{-1}(x)$ generates a matrix by reordering the elements of $x$ into columns.
} 


$$
+\sum_{N=\bar{N}}^{\infty} p_{0}^{N-1} \sum_{i=1}^{r} p_{i} F_{\bar{N}, i} \bar{A}^{N} Y\left(\bar{A}^{N}\right)^{T} F_{\bar{N}, i}^{T} .
$$

Suppose that there exists $\bar{Y} \succ 0$ such that $\mathfrak{L}(\bar{Y}) \prec \bar{Y}$. Then,

a) For all $W \succeq 0, \lim _{k \rightarrow \infty} \mathfrak{L}^{k}(W)=0$.

b) Let $U \succeq 0$ and consider the linear system $Y_{k+1}=\mathfrak{L}\left(Y_{k}\right)+U$, initialized at $Y_{0}$, then the sequence $\left\{Y_{k}\right\}$ is bounded.

Theorem 3.3. Suppose that the set $\mathcal{L}$ in (17) solves problem (24), i.e., exists $\bar{P} \succ 0$ such that $\mathfrak{E}\{\bar{P}\} \preceq \bar{P}$. Then, for any initial condition $P_{0} \succeq 0$ the sequence $\left\{P_{k}\right\}$ is bounded, i.e., $\left\{P_{k}\right\} \preceq M_{P}$.

Proof. Considering the linear operator on Lemma 3.1, we have $\mathfrak{L}(\bar{P}) \prec \mathfrak{E}\{\bar{P}\} \preceq \bar{P}$. Thus, $\mathfrak{L}$ meets the condition of Lemma 3.1. The evolution of $P_{k}$ is expressed as $P_{k+1}=\mathfrak{E}\left\{P_{k}\right\}=\mathfrak{L}\left(P_{k}\right)+U$. Since $U$ contains the disturbance and noise covariance (both positive definite), then $U \succ 0$, leading that $\left\{P_{k}\right\}$ is bounded.

Theorem 3.4. Suppose that the set $\mathcal{L}$ in (17) solves problem (24). Then, for any initial condition $P_{0} \succeq 0$, the iteration $P_{k+1}=\mathfrak{E}\left\{P_{k}\right\}$ converges to the unique positive semi-definite solution $\bar{P}$ obtained in problem (24), i.e., $\lim _{k \rightarrow \infty} P_{k}=\lim _{k \rightarrow \infty} \mathfrak{E}^{k}\left\{P_{0}\right\}=\bar{P} \succeq 0$, where $\bar{P}=\mathfrak{E}\{\bar{P}\}$

Proof. First, let us show the convergence of sequence $\left\{P_{k}\right\}$ with initial value $Q_{0}=0$. Let $Q_{k}=\mathfrak{E}\left\{Q_{k-1}\right\}=\mathfrak{E}^{k}\left\{Q_{0}\right\}$, then from (18), $Q_{1} \succeq Q_{0}=0$ and $Q_{1}=\mathfrak{E}\left\{Q_{0}\right\} \preceq \mathfrak{E}\left\{Q_{1}\right\}=Q_{2}$. By induction, $\left\{Q_{k}\right\}$ is non decreasing. Also, by Lemma 3.1, $\left\{Q_{k}\right\}$ is bounded and by Theorem 3.3 there exists an $M_{Q_{0}}$ such that $Q_{k} \preceq M_{Q_{0}}$ for any $k$. Hence, the sequence converges and $\lim _{k \rightarrow \infty} Q_{k}=\bar{P} \succeq 0$, where $\bar{P}$ is a fixed point, i.e, $\bar{P}=\mathfrak{E}\{\bar{P}\}$.

Second, we state the convergence of $G_{k}=\mathfrak{E}^{k}\left\{G_{0}\right\}$, initialized at $G_{0} \succeq \bar{P}$. Since $G_{1}=\mathfrak{E}\left\{G_{0}\right\} \succeq$ $\mathfrak{E}\{\bar{P}\}=\bar{P}$, then $G_{k} \succeq \bar{P}$ for any $k$. Moreover

$$
0 \preceq G_{k+1}-\bar{P}=\mathfrak{E}\left\{G_{k}\right\}-\mathfrak{E}\{\bar{P}\}=\mathfrak{L}\left(G_{k}-\bar{P}\right) .
$$

As $G_{k}-\bar{P} \succeq 0$, following the results on Lemma 3.1 , then $0 \preceq \lim _{k \rightarrow \infty}\left(G_{k}-\bar{P}\right)=0$, i.e., the sequence $\left\{G_{k}\right\}$ converges to $\bar{P}$.

We demonstrate now that for any initial condition $P_{0} \succeq 0$, the iteration $P_{k}=\mathfrak{E}\left\{P_{k-1}\right\}$ converges to $\bar{P}$. Since $0 \preceq Q_{0} \preceq P_{0} \preceq G_{0}$, we derive by induction that $0 \preceq Q_{k} \preceq P_{k} \preceq G_{k}$. Therefore, as $\left\{Q_{k}\right\}$ and $\left\{G_{k}\right\}$ converges to $\bar{P}$, then $\left\{P_{k}\right\}$ also converges to $\bar{P}$ and the convergence is demonstrated.

Finally, we demonstrate that

$$
\bar{P}=\arg \min _{P} \operatorname{tr}(P) \text { subject to }(25),(26)
$$

Suppose this is not true, i.e. $\hat{P}$ solves the optimization problem but $\hat{P} \neq \mathfrak{E}\{\hat{P}\}$. Since $\hat{P}$ is a feasible solution, then $\hat{P} \succ \mathfrak{E}\{\hat{P}\}=\hat{\hat{P}}$. However, this implies $\operatorname{tr}(\hat{P})>\operatorname{tr}(\hat{\hat{P}})$, which contradicts the hypothesis of optimality of matrix $\hat{P}$. Therefore $\hat{P}=\mathfrak{E}\{\hat{P}\}$. Furthermore $\bar{P}$ is unique since for a set of observer gains such that

$$
[\bar{P}, \mathcal{L}]=\arg \min _{P, \mathcal{L}} \operatorname{tr}(P) \text { subject to }(25),(26)
$$

we have shown that the sequence converges to $\bar{P}$, and this concludes the theorem.

Problem (24) minimizes the expected value of the state estimation error covariance matrix at the updating periods $t_{k}$. However, a more representative measure of the estimation performance 
is the value of the covariance matrix at each control period $t$. The following theorem allow us to obtain this value.

Theorem 3.5. Given $P=\mathcal{E}\left\{\tilde{x}_{k} \tilde{x}_{k}^{T}\right\}$, the covariance matrix of the estimation error at each period is given by

$$
\begin{gathered}
P_{t}=\mathcal{E}\left\{\tilde{x}[t] \tilde{x}[t]^{T}\right\}=\left(1-p_{0}\right) P+ \\
p_{0} \operatorname{vec}^{-1}\left(\left(I-p_{0} \bar{A} \otimes \bar{A}\right)^{-1} \operatorname{vec}\left(S_{c}\right)\right)
\end{gathered}
$$

where $S_{c}=\left(\bar{A} P \bar{A}^{T}+\left(1-p_{0}\right)^{-1} \bar{B}_{w} W \bar{B}_{w}^{T}\right)$.

Proof. The expected value of the covariance matrix of the estimation error at the control period $\left(\mathcal{E}\left\{\tilde{x}[t] \tilde{x}[t]^{T}\right\}\right)$ is computed $P$ as

$$
\begin{gathered}
\left(1-p_{0}\right) P+\sum_{N=1}^{\infty} p_{0}^{N}\left(\bar{A}^{N} P\left(\bar{A}^{N}\right)^{T}+\sum_{i=1}^{N} \bar{A}^{i-1} \bar{B}_{w} W \bar{B}_{w}^{T}\left(\bar{A}^{i-1}\right)^{T}\right) \\
=\left(1-p_{0}\right) P+\sum_{N=1}^{\infty} p_{0}^{N} \bar{A}^{N} P\left(\bar{A}^{N}\right)^{T} \\
+p_{0}\left(\sum_{i=0}^{\infty} p_{0}^{i}\right) \sum_{j=0}^{\infty} p_{0}^{j} \bar{A}^{j} \bar{B}_{w} W \bar{B}_{w}^{T}\left(\bar{A}^{j}\right)^{T},
\end{gathered}
$$

where the infinite addends can be written respectively as

$$
\begin{aligned}
& \operatorname{vec}^{-1}\left(\left(I-p_{0} \bar{A} \otimes \bar{A}\right) \operatorname{vec}\left(p_{0} \bar{A} P \bar{A}^{T}\right)\right) \\
& \operatorname{vec}^{-1}\left(\left(I-p_{0} \bar{A} \otimes \bar{A}\right) \operatorname{vec}\left(\bar{B}_{w} W \bar{B}_{w}^{T}\right)\right)
\end{aligned}
$$

Then, it finally leads to (28).

Remark 3.1. Matrix $P_{t}=\mathcal{E}\left\{\tilde{x}[t] \tilde{x}[t]^{T}\right\}$ is a linear combination of $P=\mathcal{E}\left\{\tilde{x}\left[t_{k}\right] \tilde{x}\left[t_{k}\right]^{T}\right\}$. We refer to the right hand of (28) as a linear operator $\mathfrak{F}\{\}$ that applies to $P$ as $P_{t}=\mathfrak{F}\{P\}$. The set of observer gains $\mathcal{L}$ obtained as the solution of the optimization problem

$$
\min \operatorname{tr}(\mathfrak{F}\{P\}), \quad \text { s.t. }(25),(26),
$$

minimizes the expected value of the covariance matrix at each control period (with or without measurements).

\section{Numerical computation}

The optimization problem (27) is a nonconvex optimization problem because of the terms $Q=$ $P^{-1}$ and $R=S_{P}^{-1}$. One approach to obtain the solution of this problem is the reformulation as a rank-constrained LMI problem, leading to constraints

$$
\operatorname{rank}\left(\left[\begin{array}{cc}
S_{P}-p_{0} \bar{A} S_{P} \bar{A}^{T} & I \\
I & Q
\end{array}\right]\right) \leq \bar{n}, \operatorname{rank}\left(\left[\begin{array}{cc}
R & I \\
I & S_{P}
\end{array}\right]\right) \leq \bar{n}
$$

In this work, we address the rank-constrained LMI problem (27) with a cone complementarity linearization algorithm (CCL) El Ghaoui et al. (1997), leading to

$$
\min \operatorname{tr}\left(P Q+S_{P} R\right)
$$




$$
\text { s.t. }(25), \operatorname{tr}(P)<\gamma,\left[\begin{array}{cc}
P & I \\
I & Q
\end{array}\right] \succeq 0,\left[\begin{array}{cc}
S_{P} & I \\
I & R
\end{array}\right] \succeq 0
$$

where $S_{P}$ is the matrix defined in (19) and $\gamma$ is a real positive value. Condition $\operatorname{tr}(P)<\gamma$ can be replaced by $\operatorname{tr}(\mathfrak{F}\{P\})<\gamma$ to minimize the expected value of the covariance matrix at control periods (see Remark 3.1). We solve the nonlinear minimization problem with a bisection algorithm over the CCL as follows.

Step 1 Choose a large enough initial $\gamma_{u}$ such that there exists a feasible solution to LMI conditions (25), (32) with $\gamma=\gamma_{u}$. Set $\gamma_{l}=0$, and $\gamma=\frac{1}{2}\left(\gamma_{l}+\gamma_{u}\right)$.

Step 2 Set $k=0$ and find a feasible solution set $\left[P^{0}, Q^{0}, S_{P}^{0}, R^{0}, \mathcal{L}^{0}\right]$, satisfying $(25),(32)$.

Step 3 Solve the following LMI problem for the decision variables $P, Q, S_{P}, R$ and $\mathcal{L}$ :

$$
\begin{aligned}
& \min \operatorname{tr}\left(P^{k} Q+Q^{k} P+S_{P}^{k} R+R^{k} S_{P}\right) \\
& \text { subject to }(25),(32) \\
& \text { set } k=k+1,\left[P^{k}, Q^{k}, S_{P}^{k}, R^{k}\right]=\left[P, Q, S_{P}, R\right] .
\end{aligned}
$$

Step 4 If $k<k_{\max }$ for a given prescribed maximum number of iterations $k_{\max }$, and (25) is unsatisfied after replacing $Q$ by $P^{-1}$ and $R$ by $S_{P}^{-1}$, then return to Step 3. If $k<k_{\max }$ and (25) are satisfied, update the upper bound on $\gamma$ as $\gamma_{u}=\gamma$, store the actual observer gains $L_{N, i}$, and go to Step 5. If $k=k_{\max }$, update the lower bound on $\gamma$ as $\gamma_{l}=\gamma$ and go to Step 5.

Step 5 If $\gamma_{u}-\gamma_{l} \geq \delta$, for a given small $\delta$, update $\gamma$ with $\gamma=\frac{1}{2}\left(\gamma_{l}+\gamma_{u}\right)$ and go to Step 2. If $\gamma_{u}-\gamma_{l}<\delta$ exit with the last stored set of gains $\mathcal{L}$ in Step 4 .

\section{Complexity reduction}

The solution of the previous section leads to a number of stored matrices equal to ${ }^{4}|\mathcal{L}|=\bar{N} \cdot r$, each one used for a different pair $\left(N, \eta_{i}\right)$. We can reduce the complexity of the observer in terms of storage choosing a small $\bar{N}$ and imposing some equality constraints over the set $\mathcal{L}$ as $L_{N, i}=L_{N, j}$. Problem (27) allows to easily include equality constraints over the set $\mathcal{L}$, and the choice of $\bar{N}$ only affects on the construction of the matrices of the LMI problem. Reducing the number of gains also simplifies the numerical burden of (27), as the number of decision variables and the size of the matrices are decreased. Sharing gains has also implications on the implementation of the selection mechanism. In the aim of implementing an observer with a simple online lookingup-table procedure and low storage, we propose the following preconfigured sets of equalities over the possible sensor availability combinations. The gain selection mechanism is mainly based on counting the number of available sensors and the number of consecutive periods without data.

- S1. The observer gains depend only on the intersample periods, leading to $\left|\mathcal{L}_{S 1}\right|=\bar{N}$, i.e., $L_{N, i}=L_{N, j}$ for any pair $i \neq j$.

- S2. The observer gains depend on the number of real sensors from which measurements arrive successfully at a control period, leading to $\left|\mathcal{L}_{S 2}\right|=n_{m} \cdot \bar{N}$.

- S3. The observer gains depend on the number of real and fictitious sensors from which measurements arrive successfully at a control period, leading to $\left|\mathcal{L}_{S 3}\right|=\bar{n}_{m} \cdot \bar{N}$.

- S4. The observer gains are different depending on the sampling scenario (this is the general case), leading to $\left|\mathcal{L}_{S 4}\right|=\left(2^{\bar{n}_{m}}-1\right) \cdot \bar{N}$.

The selection one of the previous gain grouping alternatives, together with the choice of $\bar{N}$, allows to define a compromise between implementation cost and performance. The lowest cost

${ }^{4}|\mathcal{L}|$ denotes the cardinal of the set $\mathcal{L}$, i.e., the number of elements of $\mathcal{L}$ 
and worst performance is obtained for S1 and a low value of $\bar{N}$, while the highest cost and best performance corresponds to $\mathrm{S} 4$ and a high value of $\bar{N}$. The examples illustrate this idea.

\section{Examples}

\subsection{Example 1}

In this example we analyze the different gain scheduling strategies proposed in Section 5, as well as the relationship between number of stored gains and achieved performance. We consider three different state matrices

$$
\begin{gathered}
A_{1}=\left[\begin{array}{cc}
0.8821 & -0.0351 \\
-0.0351 & 0.7347
\end{array}\right], A_{2}=\left[\begin{array}{cc}
0.8373 & -0.7207 \\
0.7207 & 0.8373
\end{array}\right], \\
A_{3}=\left[\begin{array}{cc}
1.6684 & 0.3197 \\
-0.1003 & 0.6782
\end{array}\right],
\end{gathered}
$$

where $A_{1}, A_{2}, A_{3}$ have real stable, complex conjugate unstable and one unstable eigenvalue, respectively. In the three cases we use

$$
\begin{gathered}
B_{u}=\left[\begin{array}{c}
-0.3359 \\
0.3466
\end{array}\right], C=\left[\begin{array}{ll}
0.5325 & 0.3987 \\
0.7258 & 0.3584
\end{array}\right] \\
B_{w}=\left[\begin{array}{ll}
0.0121 & 0.1347 \\
0.0112 & 0.0831
\end{array}\right] .
\end{gathered}
$$

and state disturbance and sensor noises with covariances

$$
W=\left[\begin{array}{cc}
0.2632 & -0.0027 \\
-0.0027 & 0.2466
\end{array}\right],\left[\begin{array}{l}
\sigma_{1}^{2} \\
\sigma_{2}^{2}
\end{array}\right]=\left[\begin{array}{l}
0.0086 \\
0.0079
\end{array}\right]
$$

The measurements are independently acquired through a communication network that induces a delay that varies from 0 to 1 periods. The amount of fictitious sensors is 4 , and the number of possible sampling scenarios is $2^{4}=16$. Table 1 details the availability probabilities that are assumed for each of the fictitious sensors in each example. They are defined such that the problem (31) is feasible.

Table 1. Probabilities assignment.

\begin{tabular}{|c|c|c|c|}
\hline sensor & delay & $\beta_{A_{1}, A_{2}}$ & $\beta_{A_{3}}$ \\
\hline 1 & 0 & 0.1391 & 0.3340 \\
1 & 1 & 0.1751 & 0.3064 \\
2 & 0 & 0.1397 & 0.3761 \\
2 & 1 & 0.1334 & 0.3403 \\
\hline
\end{tabular}

Let us first analyze the observer performance as a function of the number of stored gain matrices in the set (17), according to the scheduling approaches detailed on Section 5. Fig. 1 shows the dependency of the trace of the expected covariance matrix at control periods $P_{t}(28)$ with respect to the number of gain matrices for the four proposed scheduling approaches. We considered different intersample periods $\bar{N}=1, \ldots, 7$, leading to different number of stored gains (each point in the plot means a given value for $\bar{N}$ ). For the system with state matrix $A_{1}$ (stable with real eigenvalues), increasing the number of observer gains either by increasing $\bar{N}$ or due to the possible scheduling approaches do not improve significantly the performance, because the estimation performance $\left(\operatorname{tr}\left(P_{t}\right)\right)$ of the constant gain approach $(\mathrm{S} 1)$ is only a $1.7 \%$ worse than 
the optimal performance (with scheduling S4 and $N \rightarrow \infty, \operatorname{tr}\left(P_{t}^{\star}\right)$ ). In this case, a constant gain approach (S1) would be a good compromise between estimation performance and storage or implementation cost. For the system with state matrix $A_{2}$ (unstable with complex eigenvalues), the performance of the estimator can be increased significantly by using $\bar{N} \geq 2$ with respect to $\bar{N}=1$. The effect of using a more complex approach than $\mathrm{S} 2$ (with set $\mathrm{S} 3$ or $\mathrm{S} 4$ ) is negligible for the performance, but implies a high number of stored gains. In this case, selecting S2 and $\bar{N}=2$ (storing only 4 gains) leads to an acceptable compromise solution. For the state matrix $A_{3}$ it is better to increase the considered scenarios in the scheduling approach in terms of performance and memory storage, rather than increasing $\bar{N}$. In this case a good compromise could be to select $\mathrm{S} 3$ and $\bar{N}=1$ (storing only 4 gains).

Now, let us compare for the case of state matrix $A_{3}$ the results of the implementation of a Kalman filter algorithm adapted from Schenato (2008) and the proposed algorithm (in the cases indicated with a black filled symbol in Fig. 1(c)). Table 2 shows, for each studied case, the number of needed observer gains, the obtained trace of the covariance matrix for the state estimation error at the control period (through simulation) and the computational cost (upper-bound of FLOPs per iteration). The Kalman filter gives the best performance but needs almost fifteen times more operations than the proposed algorithm, with a slight improvement in performance. Regarding the proposed approach, it can be noticed that increasing the number of gains improves the performance (getting closer to the Kalman one) but the storage requirement is also increased. Furthermore, the numerical values for $\operatorname{tr}\left(P_{t}\right)$ obtained from simulations (shown in Table 2) converge to the optimal values presented graphically in Fig. 1(c).

Case S4 with $\bar{N}=1$ corresponds to the same approach presented in Smith and Seiler (2003) with no history loss. However, adding only one period of history to improve the estimation performance of Smith and Seiler (2003) implies the use of $15 \cdot 2^{4 \cdot(2-1)}=240$ different gains. Our approach needs to store only 60 gains to achieve a performance, $\operatorname{tr}\left(P_{t}\right)$, just a $6 \%$ higher than the one obtained with the Kalman filter, and just 8 gains to perform an $18 \%$ worse (Table 2).

This example shows a compromise between the estimation performance, storage and scheduling complexity. The designer can use this information to decide a maximum number of observer gains to be stored and then to chose its dependency either on the intersample period or on the possible scenarios to minimize the estimation error.

Table 2. Observers comparison for system $A_{3}$.

\begin{tabular}{|l|c|c|c|c|c|c|}
\hline Case & S1 & S2 & S3 & S3 & S4 & Kalman \\
\hline$|\mathcal{L}|$ & 1 & 2 & 4 & 8 & 60 & - \\
$\operatorname{tr}\left(P_{t}\right)$ & 0.1585 & 0.0781 & 0.0553 & 0.0498 & 0.0451 & 0.0423 \\
FLOP & 64 & 64 & 64 & 64 & 64 & 976 \\
\hline
\end{tabular}

\subsection{Example 2}

This example analyzes the influence of parameter $\bar{d}$ on the estimation performance and on the computational cost. Let us consider the system defined by $A_{1}$ and that only measurements from the first sensor are available. We assume that the probability of having a measurement available from the first sensor with a given delay, i.e $\beta_{j}=\operatorname{Pr}\left\{\alpha_{j}=1\right\}, \forall j=1, \ldots, \infty$, is given by a negative binomial distribution where the probability of success is 0.5 and the number of failures is 3. The assumed probability distribution of the network induced delays is shown in Fig. 2. Only the values for $d<20$ have been plotted as the probability of higher delays is negligible.

Fig. 3 shows the values of $\operatorname{tr}\left(P_{t}\right)$ (defined in (28)), the number of FLOPs per period (presented in Section 2) and the values of the probability of having no measurement available $p_{0}$ (defined in (14)), for different choices of $\bar{d}$ in the set $\{0,1, \ldots, 6\}$ within case S4. Choosing a higher $\bar{d}$ reduces $p_{0}$ and leads to a better performance $\left(\operatorname{tr}\left(P_{t}\right)\right.$ is lower). However, the order of the observer increases with $\bar{d}$ as well as the computational effort to obtain a solution for problem (31) and the number of FLOPs used in the online implementation. Moreover, at a given point incrementing 


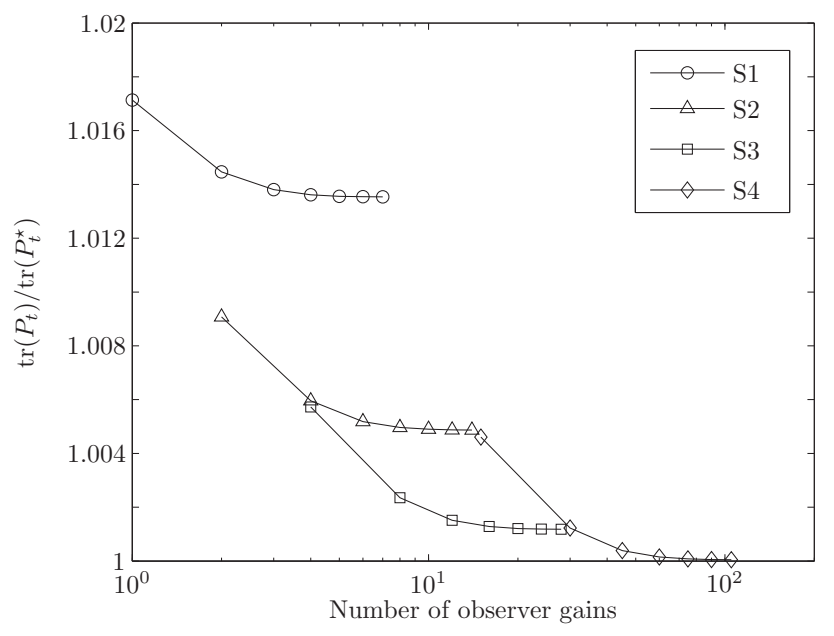

(a) Case of two real stable poles, $A_{1}$.

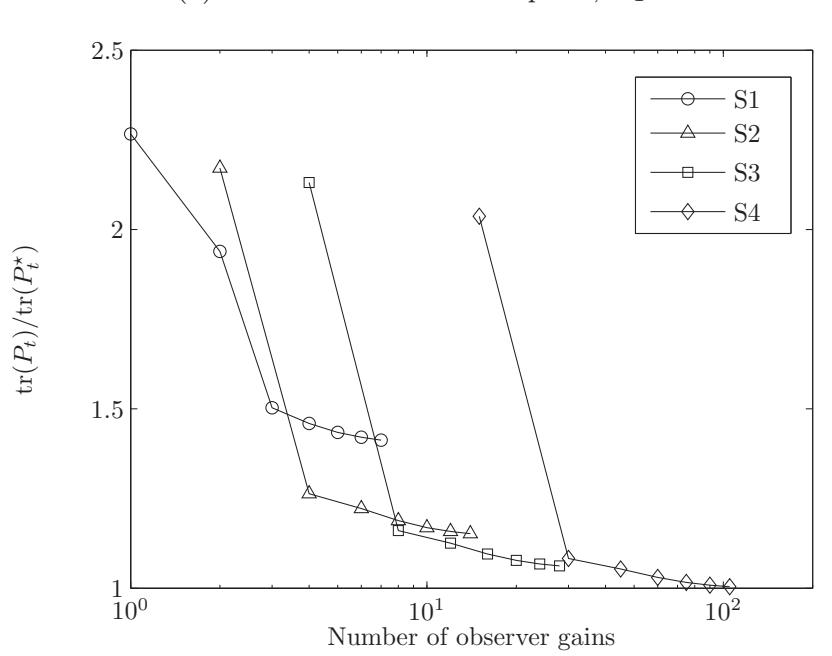

(b) Case of two complex conjugates unstable poles, $A_{2}$.

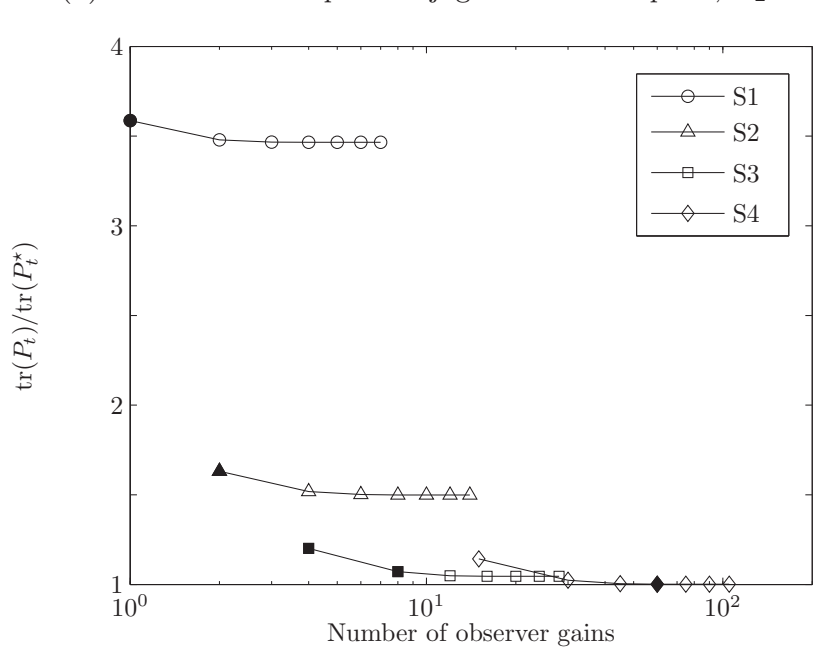

(c) Case of one unstable pole, $A_{3}$.

Figure 1. Quotient between the trace of the expected value of the estimation error covariance matrix at each control period $\operatorname{tr}\left(P_{t}\right)$ and the minimum reachable value (with scheduling S4 and $N \rightarrow \infty, \operatorname{tr}\left(P_{t}^{\star}\right)$ ) for different number of observer gains and for different scheduling approaches.

and fiferent scheduling approches.

- 
the value of $\bar{d}$ does not significantly improve the estimation performance. Therefore, parameter $\bar{d}$ must be chosen as a trade-off between estimation performance and observer complexity (number of FLOPs) plus computational effort to solve the optimization problem.

For the examined example, the case $\bar{d}=4$ could be a good choice if there is enough online computational capability available because the obtained estimation performance is only $7 \%$ worst than the optimal (with $\bar{d} \rightarrow \infty$ ). If the number of FLOPs is an important constraint, the cases with $\bar{d}=3$ and $\bar{d}=2$ reduce the number of FLOPS in a $29 \%$ and $53 \%$ respectively at the expense of increasing the estimation error to a $20 \%$ and $51 \%$ above the optimum.

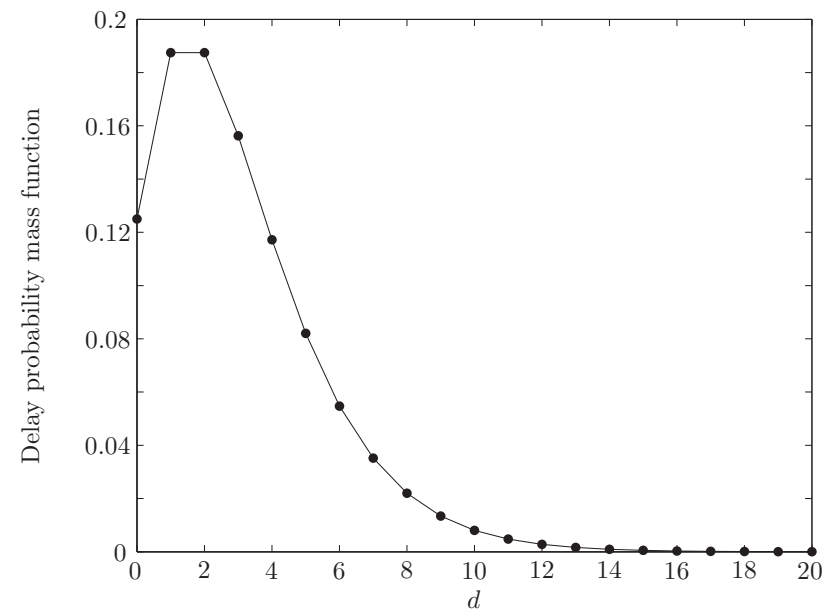

Figure 2. Probability distribution of the network induced delays, which follows a negative binomial distribution where the probability of success is 0.5 and the number of failures is 3 .
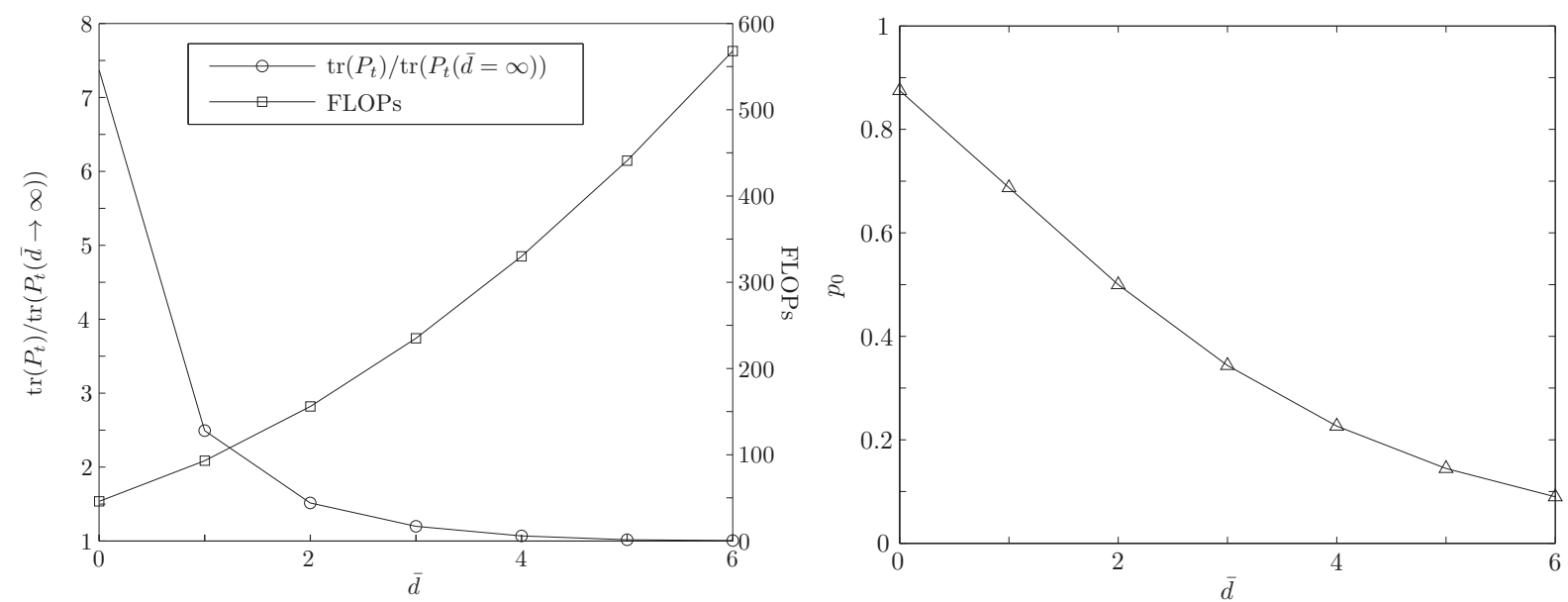

Figure 3. Normalized trace of the expected value of the estimation error covariance matrix at each control period $\operatorname{tr}\left(P_{t}\right)$, number of FLOPs, and probability of having no measurement available $p_{0}$ for different maximum considered delay $\bar{d}$.

\subsection{Example 3}

In this example, we design the observer assuming the application of the buffer approach presented in Schenato (2008) and explained in Remark 2.2. Let us consider the detectable and unstable system defined by $A_{3}$ in the first example. Let us assume that each sensor has a probability of sending a buffered packet (with the actual and the last $\bar{b}$ measurements) of $\beta_{1}=0.66$ and 
$\beta_{2}=0.11$, respectively, but when the packet is received, the delay is 0 . A parameter $\bar{d}=\bar{b}$ is selected in this case, to take into account all the measurements present in the received packets. The value of $\operatorname{tr}\left(P_{t}\right)$ decreases when the buffer is enlarged (with scheduling approach $\mathrm{S} 4$ ), and therefore the estimation is improved (Fig. 4). The buffer approach allows to reduce the use of the

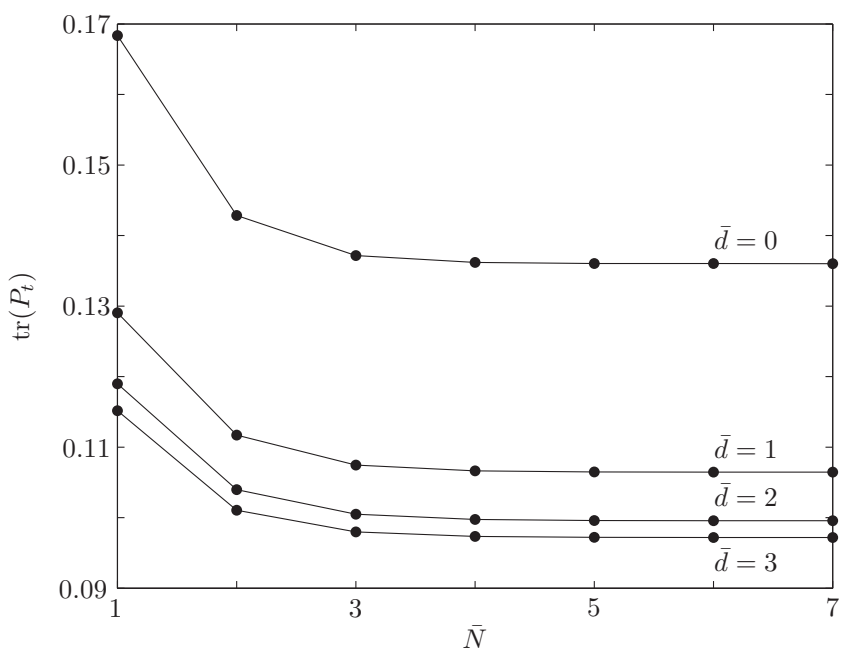

Figure 4. Trace of the expected value of the estimation error covariance matrix at each control period $\operatorname{tr}\left(P_{t}\right)$ as a function of the maximum intersample period $\bar{N}$ (with S4) for different buffer lengths $\bar{b}$.

network. To illustrate this phenomena, let us consider that only the first sensor of the considered systems is available and that we want a performance $\operatorname{tr}\left(P_{t}\right) \leq 0.03$, with the lowest network resources (measured as the probability of available measurement). We search for the minimum probability (network usage) with different buffer lengths $\bar{b}$ and different intersampling periods $\bar{N}$. The achievable performance varies with each strategy and with the value of probability $\beta_{1}$ (see Fig. 5, with approach S4). Thus, for $\operatorname{tr}\left(P_{t}\right)=0.03$, the probability of having available the measurement from the sensor can be decreased in a $12 \%$ from the worst case $(\bar{N}=1, \bar{b}=0$, $\left.\beta_{1}=0.9730\right)$ to the best one $\left(\bar{N}=4, \bar{b}=3, \beta_{1}=0.8530\right)$.
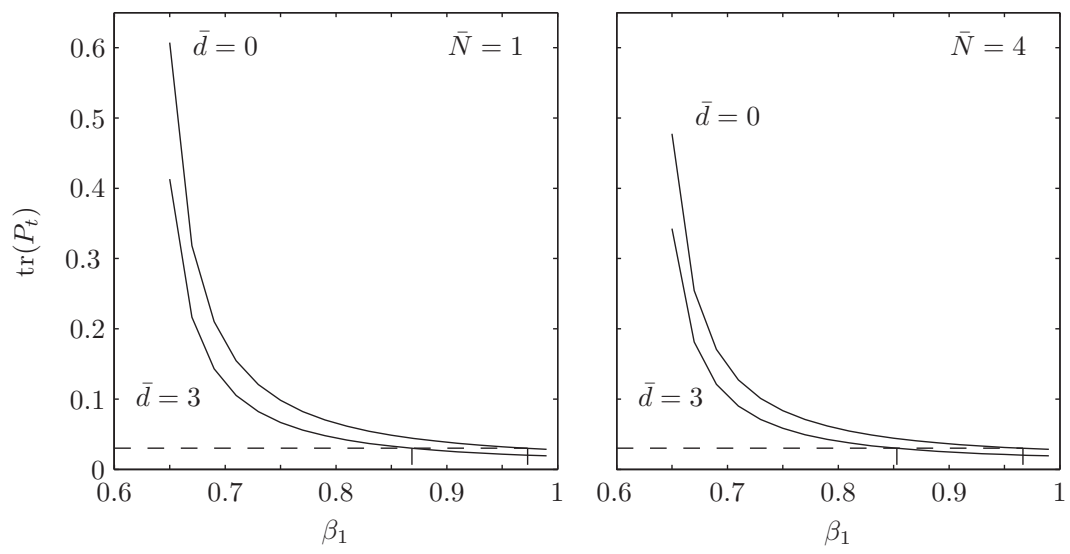

Figure 5. Trace of the expected value of the estimation error covariance matrix at each control period $\operatorname{tr}\left(P_{t}\right)($ with $\mathrm{S} 4)$ as a function of the probability of having available the measurement for different buffer lengths $(\bar{d}=0$ and $\bar{d}=3)$ and different intersample periods $(\bar{N}=1$ and $\bar{N}=4)$. 


\section{Conclusions}

In this work we designed a jump state estimator for networked control systems where the complexity in terms of storage requirements and selection mechanism is a design parameter, and we quantified the degradation that comes with the reduction of the observer complexity. The proposed approach allows to find a compromise between online computational implementation cost and performance. The result is a finite set of gains that are applied depending on the number of consecutive periods without measurements and on the available measurements. We modeled the sampling scenario due to the network through the probabilities associated to the successful reception of each sample. As a consequence, the number of control periods between consecutive measurements, $N_{k}$, is unbounded, and the associated delays are time-varying. We used a model that accepts out-of-sequence measurements, buffered measurements on a single packet or multi-rate sensor measurements. The computational cost of the on-line implementation is lower than the Kalman filter approaches with extended model, while the achieved performance is close to that one. The performance is better than the constant gain approaches at the cost of more storage requirements, and is similar to the one obtained with finite history loss jump estimators, while requiring less storage. Our approach requires additional work to become applicable under Markov chain models of the missing data, and further research must be done to determine a priori the feasibility of the LMI problem and the degradation for the use of common gains and for the choice of $\bar{N}$ and $\bar{d}$.

\section{Acknowledgement}

This work has been funded by project DPI2011-27845-C02-02 and by Beca predoctoral per a la formació de personal investigador, Pla de promoció de la investigació de la Universitat Jaume I 2011 PREDOC/2011/37

\section{References}

El Ghaoui, L., Oustry, F., and AitRami, M. (1997), "A cone complementarity linearization algorithm for static output-feedback and related problems," IEEE Transactions on Automatic Control, 42(8), $1171-1176$.

Fletcher, A., Rangan, S., Goyal, V., and Ramchandran, K. (2006), "Causal and Strictly Causal Estimation for Jump Linear Systems: An LMI Analysis," in Information Sciences and Systems, 2006 40th Annual Conference on, march, Princeton, NJ, pp. 1302-1307.

Gupta, R.A., and Chow, M.Y. (2010), "Networked Control System: Overview and Research Trends," IEEE Transactions on Industrial Electronics, 57(7), 2527-2535.

Jia, T., Niu, Y., and Zou, Y. (2012), "Sliding mode control for stochastic systems subject to packet losses," Information Sciences, 217(June), 117-126.

Liang, Y., Chen, T., and Pan, Q. (2010), "Multi-rate stochastic $\mathcal{H}_{\infty}$ filtering for networked multi-sensor fusion," Automatica, 46(2), 437-444.

Moayedi, M., Foo, Y.K., and Soh, Y.C. (2011), "Filtering for networked control systems with single/multiple measurement packets subject to multiple-step measurement delays and multiple packet dropouts," International Journal of Systems Science, 42(3), 335-348.

Peñarrocha, I., Dolz, D., and Sanchis, R. (2014), "Inferential networked control with accessibility constraints in both the sensor and actuator channels," International Journal of Systems Science, 45(5), 1180-1195.

Peñarrocha, I., Sanchis, R., and Albertos, P. (2012), "Estimation in multisensor networked systems with scarce measurements and time varying delays," Systems 83 Control Letters, 61(4), 555-562.

Sahebsara, M., Chen, T., and Shah, S.L. (2007), "Optimal $\mathcal{H}_{2}$ filtering in networked control systems with multiple packet dropout," IEEE Transactions on Automatic Control, 52(8), 1508-1513. 
Sanchis, R., Peñarrocha, I., and Albertos, P. (2007), "Design of robust output predictors under scarce measurements with time-varying delays," Automatica, 43(2), 281-289.

Schenato, L. (2007), "Optimal sensor fusion for distributed sensors subject to random delay and packet loss," 46th IEEE Conference on Decision and Control, pp. 1547-1552.

Schenato, L. (2008), "Optimal estimation in networked control systems subject to random delay and packet drop," IEEE Transactions on Automatic Control, 53(5), 1311-1317.

Sinopoli, B., Schenato, L., Franceschetti, M., Poolla, K., Jordan, M.I., and Sastry, S.S. (2004), "Kalman Filtering with Intermittent Observations," IEEE Transactions on Automatic Control, 49(9), 1453-1464.

Smith, S.C., and Seiler, P. (2003), "Estimation With Lossy Measurements : Jump Estimators for Jump Systems," IEEE Transactions on Automatic Control, 48(12), 2163-2171.

Wang, Z., Shen, B., and Liu, X. (2012), "H ${ }_{\infty}$ Filtering With Randomly Occurring Sensor Saturations and Missing Measurements," Automatica, 48(3), 556-562.

Wu, Y.L., Shen, Z.P., and Liu, Y.Y. (2012), "Mean square detectability of multi-output systems over stochastic multiplicative channels," IET Control Theory $\&$ Applications, 6(6), 796.

Zhu, C., Xia, Y., Yan, L., and Fu, M. (2012), "Centralised fusion over unreliable networks," International Journal of Control, 85(4), 409-418. 